University of Rhode Island

DigitalCommons@URI

Mechanical, Industrial \& Systems Engineering

Faculty Publications

Mechanical, Industrial \& Systems Engineering

4-18-2014

\title{
Nonlinear System Identification and Modeling of a New Fatigue Testing Rig Based on Inertial Forces
}

\author{
Michael Falco \\ University of Rhode Island \\ Ming Liu \\ University of Rhode Island \\ Son Hai Nguyen \\ University of Rhode Island \\ David Chelidze \\ University of Rhode Island, chelidze@uri.edu \\ Follow this and additional works at: https://digitalcommons.uri.edu/mcise_facpubs
}

The University of Rhode Island Faculty have made this article openly available.

Please let us know how Open Access to this research benefits you.

This is a pre-publication author manuscript of the final, published article.

Terms of Use

This article is made available under the terms and conditions applicable towards Open Access

Policy Articles, as set forth in our Terms of Use.

\section{Citation/Publisher Attribution}

Falco, M., Liu, M., Nguyen, S. H., \& Chelidze, D. (2014). Nonlinear System Identification and Modeling of a New Fatigue Testing Rig Based on Inertial Forces. Journal of Vibration and Acoustics, 136(4), 041001. doi: 10.1115/1.4027317

Available at: http://dx.doi.org/10.1115/1.4027317

This Article is brought to you for free and open access by the Mechanical, Industrial \& Systems Engineering at DigitalCommons@URI. It has been accepted for inclusion in Mechanical, Industrial \& Systems Engineering Faculty Publications by an authorized administrator of DigitalCommons@URI. For more information, please contact digitalcommons-group@uri.edu. 


\title{
A New Fatigue Testing Rig Based on Inertial Forces
}

\author{
Michael Falco, Ming Liu, Son Hai Nguyen and David Chelidze* \\ Department of Mechanical, Industrial and Systems Engineering \\ University of Rhode Island, Kingston, RI 02881 \\ Email: chelidze@egr.uri.edu
}

\begin{abstract}
A novel fatigue testing rig based on initial force is introduced. The test rig has capacity to mimic various loading conditions including high frequency loads. The rig design allows reconfigurations to accommodate a range of specimen sizes, and changes in structural elements and instrumentation. It is designed to be used as a platform to study the interaction between fatigue crack propagation and structural dynamics. As the first step to understand this interaction, a numerical model of testing rig is constructed using nonlinear system identification approaches. Some initial testing results also are reported.
\end{abstract}

\section{Introduction}

Material fatigue has been a subject of interest since the 1840's. Pioneered by Wilhelm Albert [1], scientists and engineers use fatigue testing rigs as powerful tools to validate theories and mechanical designs, and fatigue tests have become a critical procedure for manufacturing industry $[2,3]$. To be competitive in the modern market, additional requirements are posed: fatigue tests have to be conducted quickly and able to fit into fast product development cycles; and the tests have to reflect real load scenario, so over-design can be avoided without damaging reliability.

Traditional fatigue testing rigs have difficulty to meet both of the requirements at the same time. Based on methods of load application, the testing rigs can be classified into $[4,5]: 1)$ spring force, 2) centrifugal force, 3 ) hydraulic force, 4) pneumatic force, 5) thermal dilatation force, and 6) electro-magnetic force based loads. Among these rigs, 1), 2 ), and 6) usually can only provide periodic loads; and 3), 4), and 5) are limited by their low frequency load application. Although literature reviews also showed fatigue testing machines with ultrahigh excitation frequency [6], these rigs were designed for tiny components which were beyond the range of discussion here.

A novel fatigue testing rig based on inertial forces was designed and constructed at the University of Rhode Island's

*Address all correspondence to this author.
Nonlinear Dynamics Laboratory. This new rig can duplicate arbitrary load histories with a high testing speed (30 $\mathrm{Hz}$ or above), at the same time, it permitted fatigue tests at various $R$-ratios ${ }^{1}$ (including zero or negative), which can be controlled accurately and is adjustable during a test. This work was motivated by similar testing rig [7]. However, our horizontal - in contrast with their vertical - arrangement allows for more range in loading rates and frequencies, and facilitates reconfigurable test design. Our system can be easily reconfigured to accommodate range of different specimens, as well as changes to other structural elements and sensing instrumentation.

In a mechanical structure, fatigue crack propagation is not an isolated process. Fatigue process is driven by the structural dynamics, but it also influences this dynamics by changing structural parameters. Hence, fatigue life prediction methods cannot be accurate without taking into consideration this interaction between crack propagation and structural dynamics. Based on authors' knowledge, the research about the coupling between fatigue crack propagation and structure dynamics is limited [7].

Equipped with multiple sensors and a high performance DAQ system, the fatigue testing rig can serve as a perfect platform to study the interaction between fatigue crack propagation and structure dynamics. As the first step of related research, a reliable model of the healthy structure (the rig without cracks) is constructed. This model not only describes the structure dynamics of the structure, but serves as a baseline for future modeling efforts. Due to the inherent nonlinearity of the rig, a linear model is inadequate to describe full dynamical properties of the structure accurately. Here a nonlinear model is constructed using restoring force surface (RFS) and direct parameter estimation (DPE) methodology. A linear modal is also generated for validation purpose based on modal analysis results.

To validate usefulness of this new setup, several initial tests have been conducted using this testing rig. The collected data are used to compare the Generalized Fatigue

\footnotetext{
${ }^{1} R=\sigma_{\min } / \sigma_{\max }$
} 


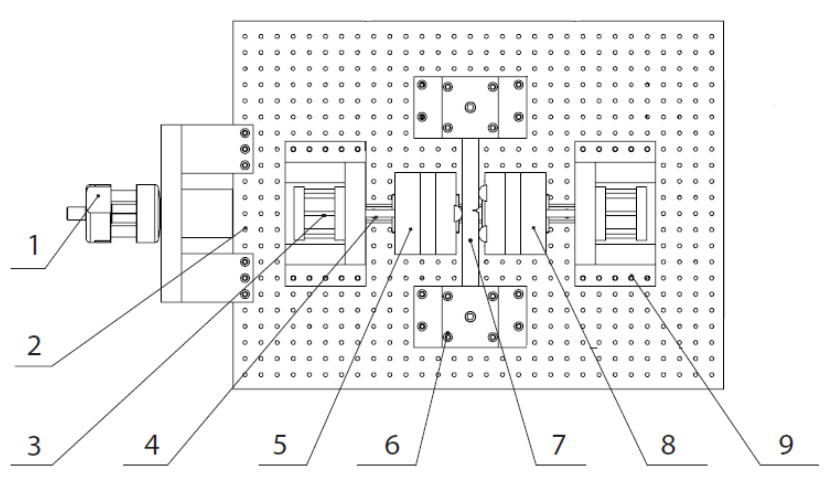

Fig. 1: Schematic of the fatigue testing apparatus. 1. Flexible connector; 2. Slip table; 3. Back cylinder; 4. Rail; 5. Back mass block; 6. Specimen supports; 7. Specimen; 8. Front mass block; 9. Front Cylinder.

Damage Coordinate's (GFDC's), calculated based on Phase Space Warping (PSW) [8-10] and Smooth Orthogonal Decomposition (SOD) [11], with direct fatigue crack measurements from and Alternating Current Potential Drop (ACPD) crack monitoring system.

In the next section the setup of the fatigue testing rig is described. Following it with the details of the nonlinear system identification, additional discussions about nonlinearity, and some initial testing results.

\section{Fatigue Testing Rig}

\subsection{Mechanical Setup}

A schematic of the testing rig is shown in Fig. 1. The mechanical backbone of the system is a slip table guided by four linear bearings on two parallel rails mounted to a granite base. An LDS electromagnetic shaker (V721 Brüel \& Kjær, German) is used to drive the slip table and mimic various loading conditions. The specimen is a single edge notched beam, which is pin-pin supported. Two inertial mass blocks, guided by linear bearings mounted to the slip table, provide dynamic loads. The masses are kept in contact with the specimen by two pneumatic cylinders. Pressures within the cylinders is set to be large enough to keep the mass blocks in contact with the specimen at all times, which allows for the transfer of inertial forces to the specimen. When the slip table moves, the specimen drives the masses to follow the motion of the slip table, and the dynamical load on the specimen is realized by the inertia force of the two mass blocks. Different $R$-ratios can be realized by adjusting the pressure in each cylinder. A photo of the system is included in Fig. 2.

The specimens are designed to follow the ASTM standard E1820-08a [12]. One of the specimens is shown in Fig. 3. The specimen is made of 6061 aluminum bar stock, with dimensions $304.8 \mathrm{~mm} \times 20.32 \mathrm{~mm} \times 12.7 \mathrm{~mm}$, and the fatigue crack is initiated by a machined ' $V$ ' notch. In order

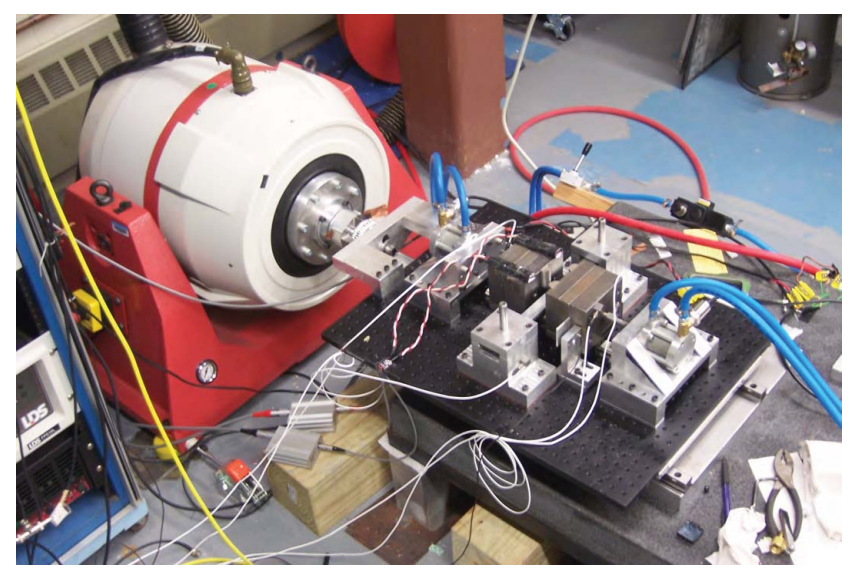

Fig. 2: Photograph of the system

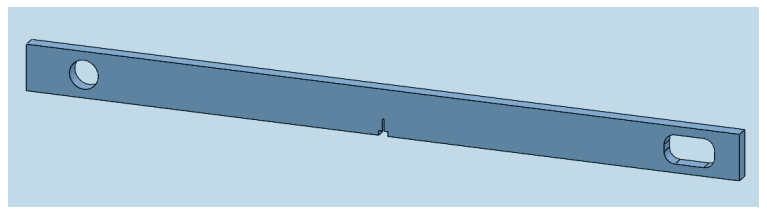

Fig. 3: Model of a specimen

to get rid of residual and machining stresses, the specimens are fully annealed prior to testing.

\subsection{Instrumentation}

The rig's control and measurement system is designed to provide excitation signals and monitor crack propagation and structural dynamics at the same time. The capacity to measure the fatigue crack growth in real time is realized by a crack growth monitor CGM-7 (Matelect, UK) based on ACPD technology. For a reliable measurement of an aluminum specimen, an alternating current is set to 0.5 Amperes at a frequency of $30 \mathrm{kHz}$. The structural response is measured using accelerometers and an eddy current displacement sensor. Two single-axis accelerometers from PCB Piezoelectronics, model number 333B42, are employed. One is attached on the slip table, and the other is attached with one mass block with both sensitive axises kept parallel with the horizontal axis. One eddy current sensor U5 with an ECL202 driver (Lion Precision), is used to measure the relative displacement between one mass block and the slip table.

Drive signals of the testing rig are generated by a program controlled function generator (Tektronix AFG 30222), which can generate different types of excitation signals based on either internal functions (such as random and harmonic) or stored arbitrary time histories (such as deterministic chaotic). The preload is measured by two pressure sensors which are directly connected to the pneumatic cylinders. The dynamical forces between the pneumatic cylinders and the masses are measured by two piezoelectric force sensors 
(Model 208C02 from PCB Piezoelectronics). All data from the sensors are recorded using a DAQ card (PCI-6013 from National Instrument) on a PC; and all control and DAQ tasks are implemented through a LabView program.

\subsection{Comparison with an existing design}

A similar inertial force based fatigue apparatus has been described in Ref. [7]. Compared with Foong's work, the new design has two major advantages. Firstly, the horizontal setup reduces the excitation force necessary to drive the system. Therefore, a similar load capacity can be achieved by using a smaller and lower cost electromagnetic shaker. Secondly, the slip table is built with a standard mounting surface, which allows for simple modifications to accommodate various types of specimens and sensing instrumentation.

\section{System Identification}

Assuming the slip table and all of the fixtures to be rigid bodies, only the dynamics of the testing structure (the mass, specimen, and pneumatic cylinders) are included in the physical model of the testing rig. Because of the nonlinear components (e.g, pneumatic cylinders), standard modal analysis [13] cannot be applied directly. Here, a nonlinear system identification procedure is utilized.

Although there is no uniform standard solution for nonlinear SID [14], the system identification task is realized through a three step process consisting of nonlinearity detection, selection of an appropriate nonlinear model, and estimation of model parameters. Each step is achieved using frequency response function overlay (FRFO), restoring force surface (RFS), and direct parameter estimation (DPE) methods, respectively.

\subsection{Detection of Nonlinearity}

The concept of FRFO is that the frequency response function (FRF) of a linear system is independent of excitation amplitude, which is not true for a nonlinear system. Therefore, nonlinearity can be detected by changes in the FRF associated with the changes in the excitation amplitude [15]. Reviews of other nonlinearity detection approach can be found in Ref. [16].

Standard modal analysis is conducted using an ACE data acquisition card and Signal Calc modal analysis software from Data Physics, Inc.. The FRF is calculated from data averaged over 16 windows of burst-random excitation. This excitation is chosen to mitigate spectral leakage. Eighty percent of the window size is dedicated to random excitation, while the remaining twenty percent is zero-level excitation. In the experiment, the input signal is from an accelerometer mounted to a rigid support, while the output signal is from an accelerometer mounted to the front mass block. Both signals are high pass filtered at $5 \mathrm{~Hz}$ in order to remove low fre-

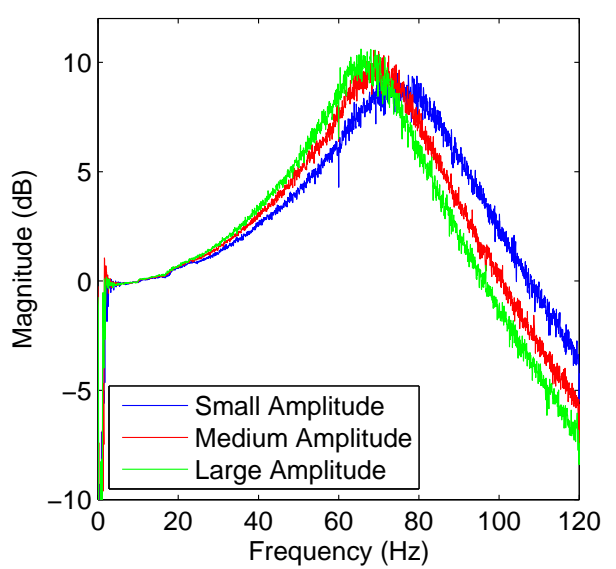

Fig. 4: Model of a specimen

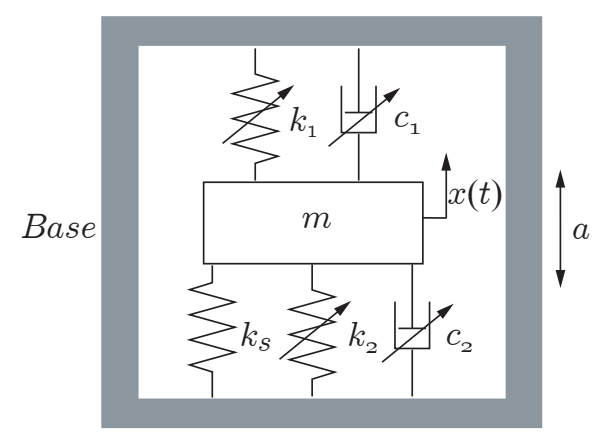

Fig. 5: A simplified model for the fatigue testing rig

quency uncertainties related to accelerometers. Three tests are conducted at amplitude levels small, medium and large. The corresponding FRF's are shown in Fig. 4. As the amplitude increases, the peaks of the FRF moves towards the lower frequency range, which is indicative of a softening spring nonlinear characteristic.

\subsection{Nonlinear Model Selection}

The major challenge in nonlinear system identification is model selection [14]. Because our testing rig is a relatively simple system, a two step procedure is adopted to determine appropriate system model. The first step is to build a simple physical model with some unknown functions. Then the RFS approach is used to determine the unknown functions and parameters.

The assumptions that the slip table and fixtures are rigid and the deformation of the specimen is relatively small ( $<$ $2 \times 10^{-4} \mathrm{~m}$ from its equilibrium position), allows the testing rig to be represented by a single degree-of-freedom massspring-damper system shown in Fig. 5. The specimen is modeled as a linear spring (with stiffness $k_{s}$ ). The pneumatic cylinders are represented by a combination of nonlinear springs $\left(k_{1}\right.$ and $\left.k_{2}\right)$ and nonlinear dampers $\left(c_{1}\right.$ and $\left.c_{2}\right)$. 
The system can be described by the differential equation in the form of:

$$
m \ddot{x}+c_{1}(x, \dot{x})+c_{2}(x, \dot{x})+k_{1}(x, \dot{x})+k_{2}(x, \dot{x})+k_{s} x=-m a,
$$

where $m$ is the total mass of the two mass blocks; $a$ is the base acceleration; $x$ is the relative displacement measured by the eddy current sensor, and dots represent time differentiation. Further simplification can be made resulting in:

$$
m \ddot{x}+C(x, \dot{x})+K(x, \dot{x})+k_{s} x=-m a,
$$

where $C(x, \dot{x})=c_{1}(x, \dot{x})+c_{2}(x, \dot{x})$ and $K(x, \dot{x})=k_{1}(x, \dot{x})+$ $k_{2}(x, \dot{x})$.

Restoring force [17] is defined as a variable force that gives rise to an equilibrium in a physical system. For a SDOF system, it can be written as:

$$
f(x, \dot{x})=F-m \ddot{x}
$$

where $F$ is the external force and $m$ is the mass. In our case, the restoring force equals:

$$
f(x, \dot{x})=-m a-m \ddot{x}=C(x, \dot{x})+K(x, \dot{x})+k_{s} x .
$$

The related RFS approach is one of the most established nonlinear system identification methods. Although the RFS method is not applied directly in this research, the RFS is constructed and used to provide valuable information for model selection. Among all the parameters, the mass is measured directly, $a$ is measured by an accelerometer, $x$ is measured by the eddy current sensor, and $\dot{x}$ and $\ddot{x}$ are calculated using finite difference method. Therefore, the RFS can be directly generated based on the collected data sets.

The restoring forces are calculated using $6 \times 10^{5}$ data points, while the testing rig is driven by a random signal with limited frequency band ( 0 to $60 \mathrm{~Hz})$. The amplitude of the excitation is chosen to ensure the availability of parameter identification data within the range of proposed experimental excitation amplitudes. The pressure in the two pneumatic cylinders are set to $15 \mathrm{psi}$. The data are recorded with DAQ frequency $1 \mathrm{Khz}$ and the distribution of sample points on the phase plane are shown on Fig. 6. Isolated points related with discrete measurements are interpolated into a continuous surface using Sibson's natural neighbor method [18], and the measured RFS is shown in Fig. 6.

The particular form of the unknown $C(x, \dot{x})$ and $K(x, \dot{x})$ could be identified by observing the two slice views of the RFS shown in Fig. 7 with $x=0$ and $\dot{x}=0$ respectively. Since it is a reasonable assumption that that $C(x, \dot{x})$ is a function of $\dot{x}$ only and $K(x, \dot{x})$ is a function of $x$ only, $f=C(\dot{x})$ when

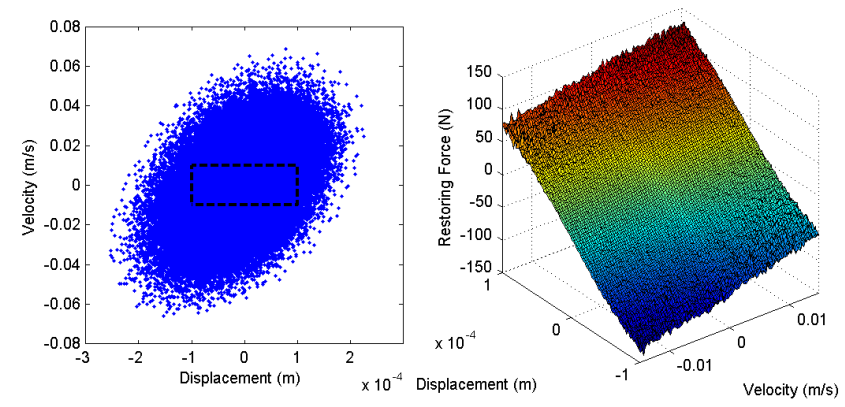

Fig. 6: Left: Distribution of data points and area used to generate RFS; Right: Generated RFS.
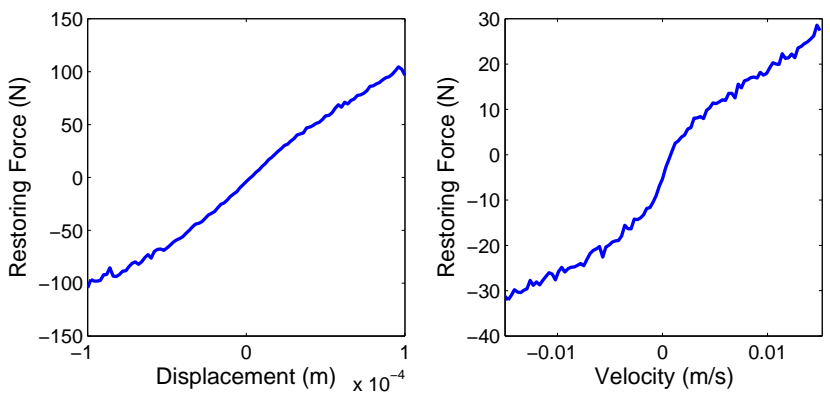

Fig. 7: Left: Slice view of RFS when $\dot{x}=0$; Right: Slice view of RFS when $x=0$.

$x=0$ and $f=K(x)+k_{s} x$ when $\dot{x}=0$ are appropriate based on these assumptions. From Fig. 7, we conclude that $C(\dot{x})$ and the $K(x)$ can be described partially by cubic polynomials. However, the changes of slope close to $\dot{x}=0$ also indicates that Coulomb damping is also present in the system.

A good experimental fit of measured damping force due to Coulomb damping [19] can be described as:

$$
F=C|\dot{x}|^{\delta_{v}} \operatorname{sgn}(\dot{x})
$$

where $F$ is the damping force and $\delta_{v}$ depends on geometry. Therefore, Eq. (2) was rewritten in the form:

$$
\begin{aligned}
m \ddot{x}+C_{f 1} \dot{x} & +C_{f 2} \dot{x}^{3}+C_{f 3}|\dot{x}|^{\alpha} \operatorname{sgn}(\dot{x}) \\
& +K_{f 1} x+K_{f 2} x^{3}+K_{f 3}|x|^{\beta} \operatorname{sgn}(x)=-m a,
\end{aligned}
$$

where $C(\dot{x})=C_{f 1} \dot{x}+C_{f 2} \dot{x}^{3}+C_{f 3}|\dot{x}|^{\alpha} \operatorname{sgn}(\dot{x})$ and $K(x)=$ $K_{f 1} x+K_{f 2} x^{3}+K_{f 3}|x|^{\beta} \operatorname{sgn}(x)$.

\subsection{Parameter Estimation}

With an assumed nonlinear model, the SID problem is solved as a parameter estimation problem. The method of DPE [20] is applied in this research. To perform DPE, Eq. 


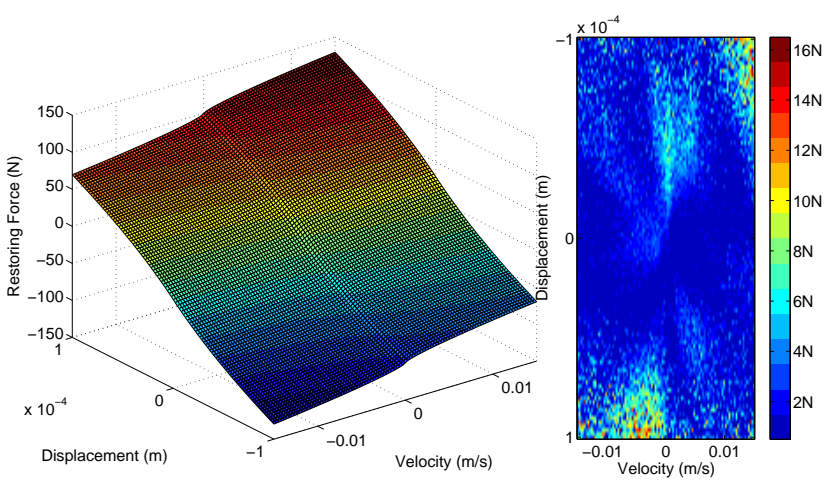

Fig. 8: Left: Surface generated using parameters from DPE; Right: The error between the modeled surface and the generated RFS.

(6) is rewritten for $n$ discrete measurements in the following form:

$$
\begin{gathered}
{\left[\begin{array}{cccccc}
\dot{x}_{1} & \dot{x}_{1}^{3} & \left.\dot{x}_{1}\right|^{\alpha} \operatorname{sgn}\left(\dot{x}_{1}\right) & x_{1} & x_{1}^{3} & \left|x_{1}\right|^{\beta} \operatorname{sgn}\left(x_{1}\right) \\
\dot{x}_{2} & \dot{x}_{2}^{3} & \left|\dot{x}_{2}\right|^{\alpha} \operatorname{sgn}\left(\dot{x}_{2}\right) & x_{2} & x_{2}^{3} & \left|x_{2}\right|^{\beta} \operatorname{sgn}\left(x_{2}\right) \\
\vdots & \vdots & \vdots & \vdots & \vdots & \vdots \\
\dot{x}_{n} \dot{x}_{n}^{3}\left|\dot{x}_{n}\right|^{\alpha} \operatorname{sgn}\left(\dot{x}_{n}\right) & x_{n} & x_{n}^{3}\left|x_{n}\right|^{\beta} \operatorname{sgn}\left(x_{n}\right)
\end{array}\right]\left[\begin{array}{c}
C_{f 1} \\
C_{f 2} \\
C_{f 3} \\
K_{f 1} \\
K_{f 2} \\
K_{f 3}
\end{array}\right]} \\
=\left[\begin{array}{cc}
\ddot{x}_{1} & a_{1} \\
\ddot{x}_{2} & a_{2} \\
\vdots & \vdots \\
\ddot{x}_{n} & a_{n}
\end{array}\right]\left[\begin{array}{l}
-m \\
-m
\end{array}\right]
\end{gathered}
$$

When $n>6$ and parameters $\alpha$ and $\beta$ are known, Eq. (7) is an over defined problem which can be solved by using a standard least squares approach. To determine the optimal $\alpha$ and $\beta$ constants, the procedure introduced in [21] is adopted. A search for optimal $\alpha \& \beta$ begins with a given step size (called grid) in a region around a pre-defined initial point (called the center) $\left[\alpha_{0}, \beta_{0}\right]$. Then the center is moved to the point $\left[\alpha_{1}, \beta_{1}\right]$ on the grid, where the least square error is minimized based on the over-defined Eq. (7). The search procedure is repeated with a half grid size. These procedures are repeated $m$ times until the value $\left|\left[\alpha_{m-1}, \beta_{m-1}\right]-\left[\alpha_{m}, \beta_{m}\right]\right|$ is smaller than a specified threshold.

The parameters are determined using the data shown in Fig. 6. Then an RFS is generated based on the selected nonlinear model and estimated parameters. The resulting surface and the error between the modeled surface and the RFS are shown in Fig. 8. The largest absolute value of error is only about $16 \mathrm{~N}$ only near the extreme displacements.

\section{Discussion and Initial Testing Results}

\subsection{Error Factors}

Although the two RFS's (based on the measured data and the model) fit each other quite well, relatively large errors are observed in the areas when the absolute value of $x$ and $\dot{x}$ are large and $x \dot{x}>0$. The errors are due to the following factors:

1. The number of sampling points in these areas is small, which leads to inaccurate estimation of the RFS from experimental data.

2. These areas represent rare dynamical states of the rig_-large displacement, velocity, acceleration, and jerk (large $|\dddot{x}|$ value), which make the numerical calculation of velocity and acceleration inaccurate.

3. With a large jerk, a time delay between different measurement channels on the DAQ system is not ignorable and causes additional errors in the calculation of acceleration.

\subsection{Dynamical Range}

There are three factors which determine the dynamical range of the rig: the maximum output force of the shaker $F_{\text {max }}=3700 \mathrm{~N}$, the allowable movement range of the shaker $D=0.0254 \mathrm{~m}$ (p-to-p), and the natural frequency of the slip table $f_{N}=150 \mathrm{~Hz}$. Because the total mass of the moving parts (the slip table and the armature moving mass) $m_{\text {total }}$ is about $45 \mathrm{~kg}$, the maximum acceleration of the slip table can be determined by $a_{\max }=F_{\max } / m_{\text {total }}=82.2 \mathrm{~m} / \mathrm{s}^{2}$ or about $8.4 \mathrm{~g}$. Unfortunately, the AC current in the lab is limited to $15 \mathrm{~A}$ (while $25 \mathrm{~A}$ is needed for full capacity). Thus, the maximum acceleration could only reach $3 \mathrm{~g}$ in a short time period. For safety reasons, the maximum acceleration is limited to $2 \mathrm{~g}$ in actual experiments.

If we assume the excitation signals are sinusoidal, the relationship between $a_{\max }, D$, and the excitation frequency $f_{e}$ can be written as: $a_{\max }=4 \pi^{2} f_{e}^{2} D / 2$. Since $D=0.0254 \mathrm{~m}$ and $a_{\max }<2 \mathrm{~g}$, the maximum acceleration could only be reached only when $f_{e}>6.3 \mathrm{~Hz}$. Because of the natural frequency of the slip table is $150 \mathrm{~Hz}$, it is reasonable to limit the excitation frequency below $30 \mathrm{~Hz}$. Hence the maximum acceleration output of the slip table can only be reached between $6.3 \mathrm{~Hz}$ and $30 \mathrm{~Hz}$.

Although increasing the mass of the blocks can improve the maximum dynamic load on the specimen, the maximum dynamic load must be smaller than the output of the pneumatic cylinders to keep the mass blocks in continuous contact with the specimen. At the same time, increasing in the mass will cause additional friction on the rails. Considering that the mass blocks contained about $20 \%$ of the $m_{\text {total }}$ already, the potential to increase the load capacity by increasing the mass of the blocks is restricted. Further improvement of the structural stiffness is also not pursued, because increasing stiffness leads to additional mass for the slip table, therefore 
the maximum load capacity decreases due to the limitation of the maximum shaker output force.

\subsection{Influence of Air Pressure}

The same system identification procedure is repeated several times under different pressures (the pressure in the two cylinders are kept identical). The estimated parameters are listed in Table 1. The relationship between the pressure and the system parameters can be described as follows: the stiffness and the damping of the testing system increase with the increase of pressure.

\subsection{Linear Region}

Although the testing rig is characterized as having cubic damping and stiffness, the nonlinearity is not strong especially when the response amplitude is relatively small. Therefore it is possible to define a small linear region in which the system can be treated linear. The definition of a linear region is also interesting since 1) there are advantages in system analysis if a linear assumption holds, and 2) a change of the linear region boundary itself can serve as an indicator for abnormality or damage.

Here non-linearity is defined by how accurately a linear model can fit the measured RFS. For a region $R_{G}$ in the phase space defined by $|x|<x_{1}$ and $|\dot{x}|<x_{2}$, a linear model is estimated based on Eq. (7) by assuming that only $C_{f 1}$ and $K_{f 1}$ are nonzero. The nonlinearity $(N)$ is quantified by:

$$
N=\frac{\max \left(\left|f(x, \dot{x})_{l}-f(x, \dot{x})_{m}\right|\right)}{\max \left(\left|f(x, \dot{x})_{m}\right|\right)},[x, \dot{x}] \in R_{G}
$$

where $f(x, \dot{x})_{l}$ is the restoring force calculated based on the linear model, and $f(x, \dot{x})_{m}$ is the measured restoring force.

Based on the above definition, the nonlinearity of different regions on the response phase space is shown in Fig. 9. For each pixel on the image, its coordinates ( $a x$ and $a y)$ define the region on the response phase space $(|x|<a x$ and $|\dot{x}|<a y)$ and its color illustrates the severity of the nonlinearity. A color bar is provided with the map to allow for convenient interpretation of the data. Nonlinearities under a $20 \mathrm{~Hz}$ sinusoidal excitation at amplitudes of $1.2 \mathrm{~g}$ and $1.6 \mathrm{~g}$ are outlined in black and blue lines respectively. Since a $1.2 \mathrm{~g}$ excitation is representative of a typical experiment (1.6g is a higher limit) and its' corresponding nonlinearity is relative low $(<15 \%)$, we can conclude that the testing rig works in a linear region under a typical load condition.

\subsection{Verification}

The restoring force surface method can be verified by comparing DPE with standard linear modal analysis approaches. As outlined previously, modal analysis can be used because it is reasonable to treat the rig as a linear system if

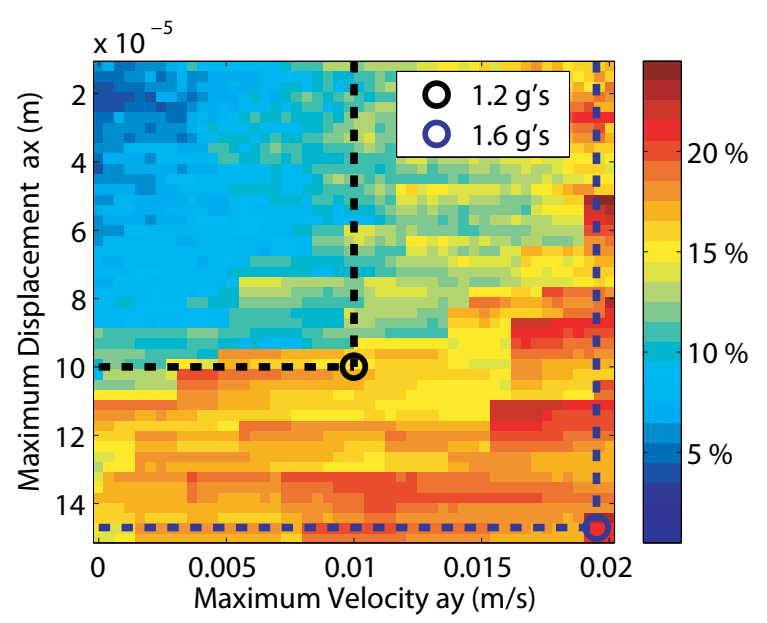

Fig. 9: Relationship between nonlinearity and vibration amplitude

the response amplitude is low. Verification of the restoring force method is conducted by comparing the results from DPE and modal analysis.

In order to directly compare the results of the two methods, short experiments are run using Signal Calc modal device. In these experiments, the response amplitude is controlled carefully, hence the linear assumption can be held (base on the results shown in Sec.4.4). The FRF data, collected using the same parameters outlined in Section 3, is fit to the known single degree of freedom FRF in the form:

$$
|G(i \omega)|=\frac{C_{s}}{\sqrt{\left[1-r^{2}\right]^{2}+(2 \zeta r)^{2}}},
$$

where $G(i \omega)$ is the frequency response, $C_{s}$ is a scaling factor, $\zeta$ is the damping ratio, and $r$ is $\omega / \omega_{n}$. The parameters, $C_{s}, \zeta$, and $\omega_{n}$ are approximated using an iterative method which minimizes the least squares error. In order to emphasize the significance of the data at areas of small velocity and displacement, a weighted least squares approach is used for DPE.

The weighting function $W(x, \dot{x})$ is a two dimensional function, which is representative of the distribution of data across the phase space $([x, \dot{x}])$. The function is normalized giving each data point a weight between zero and one, depending on the location in the phase space. Then the Eq. (7) is written as: 
Table 1: Direct Parameter Estimation Results

\begin{tabular}{||c|cccccccc||}
\hline \hline $\begin{array}{c}\text { Pressure } \\
(\mathrm{psi})\end{array}$ & $\begin{array}{c}C_{f 1} \\
(\mathrm{~N} /(\mathrm{m} / \mathrm{s}))\end{array}$ & $\begin{array}{c}C_{f 2} \\
\left(\mathrm{~N} /(\mathrm{m} / \mathrm{s})^{3}\right)\end{array}$ & $\begin{array}{c}C_{f 3} \\
\left(\mathrm{~N} /(\mathrm{m} / \mathrm{s})^{\alpha}\right)\end{array}$ & $\begin{array}{c}K_{f 1} \\
(\mathrm{~N} / \mathrm{m})\end{array}$ & $\begin{array}{c}K_{f 2} \\
\left(\mathrm{~N} / \mathrm{m}^{3}\right)\end{array}$ & $\begin{array}{c}K_{f 3} \\
\left(\mathrm{~N} / \mathrm{m}^{\beta}\right)\end{array}$ & $\alpha$ & $\beta$ \\
\hline 15 & 352.38 & $-6.17 \times 10^{4}$ & 365.17 & $1.41 \times 10^{6}$ & $8.66 \times 10^{12}$ & $-8.74 \times 10^{8}$ & 0.64 & 1.81 \\
20 & $-4.48 \times 10^{3}$ & $3.35 \times 10^{4}$ & $3.41 \times 10^{3}$ & $2.31 \times 10^{6}$ & $4.52 \times 10^{13}$ & $-7.57 \times 10^{9}$ & 0.83 & 1.93 \\
25 & $-2.46 \times 10^{3}$ & $-6.31 \times 10^{4}$ & $2.12 \times 10^{3}$ & $2.25 \times 10^{6}$ & $3.72 \times 10^{8}$ & $-1.25 \times 10^{10}$ & 0.79 & 2.00 \\
\hline \hline
\end{tabular}

$$
\begin{array}{r}
{\left[\begin{array}{cccc}
W\left(x_{1}, \dot{x}_{1}\right) & 0 & \ldots & 0 \\
0 & W\left(x_{2}, \dot{x}_{2}\right) & \ldots & 0 \\
\vdots & \vdots & \ddots & \vdots \\
0 & 0 & \ldots W\left(x_{n}, \dot{x}_{n}\right)
\end{array}\right]\left[\begin{array}{cc}
\dot{x}_{1} & x_{1} \\
\dot{x}_{2} & x_{2} \\
\vdots & \vdots \\
\dot{x}_{n} & x_{n}
\end{array}\right]\left[\begin{array}{c}
C_{f 1} \\
K_{f 1}
\end{array}\right]} \\
=\left[\begin{array}{cc}
\ddot{x}_{1} & a_{1} \\
\ddot{x}_{2} & a_{2} \\
\vdots & \vdots \\
\ddot{x}_{n} & a_{n}
\end{array}\right]\left[\begin{array}{c}
m \\
-m
\end{array}\right]
\end{array}
$$

Since the identified model is to compared with a linear model, all the nonlinear parameters, such as $\alpha, \beta$, and $C_{f 2}$ are ignored. The natural frequency is then calculated using the measured mass and the linear coefficient of stiffness $K_{f 1}$. The results are shown in Table 2.

Table 2: Comparison of estimated parameters

\begin{tabular}{ccc} 
& Modal Analysis & Restoring Force \\
\hline Pressure $(\mathrm{psi})$ & $\omega_{n}(\mathrm{rad} / \mathrm{s})$ & $\omega_{n}(\mathrm{rad} / \mathrm{s})$ \\
\hline 20 & 86.27 & 91.45 \\
25 & 88.45 & 92.11 \\
30 & 106.24 & 104.04 \\
\hline
\end{tabular}

\subsection{Initial Testing Results}

Several initial tests are conducted and results from one of the tests are shown here. A specimen is loaded for approximately 8 hours until broken ${ }^{2}$. The fatigue crack is initiated by a $7.62 \mathrm{~mm}$ notch in the center of the specimen and a

\footnotetext{
${ }^{2}$ Currently, break of a specimen is indicated by the saturation of the eddy current sensor which usually happens after a crack reaches its fracture stage.
}

(a)
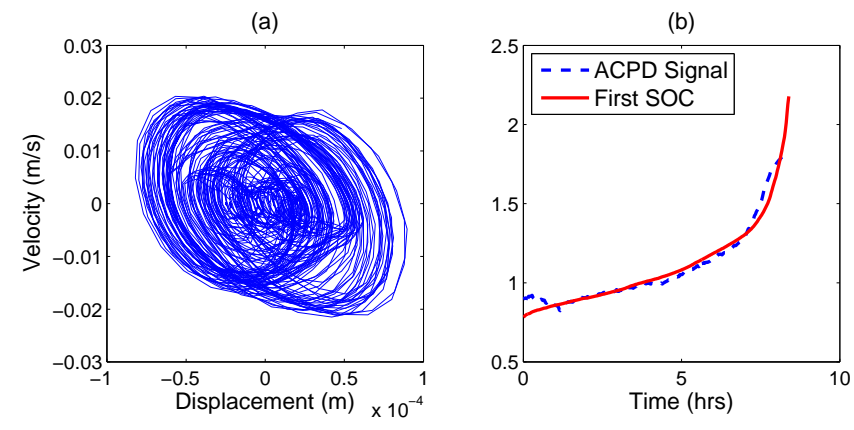

Fig. 10: (a). Phase space of the specimen under chaotic loading; (b). ACPD measurement and GFDC.

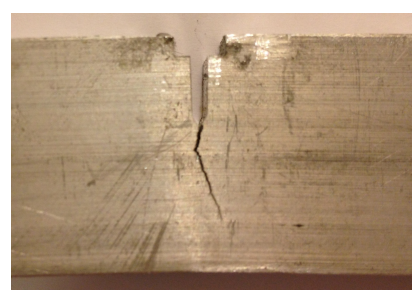

Fig. 11: Specimen at the end of the experiment

chaotic load is applied (a phase space of specimen vibration $x$ v.s. $\dot{x}$ can be seen in Fig. 10.(a)). The speciment at the end of the experiment is shown in Fig. 11

The crack propagation is tracked by the ACPD monitor. Based on the concept of PSW combined with the Smooth Orthogonal Decomposition (SOD) algorithm a GFDC is also shown to track the damage accumulation. The GFDC provided the ability to monitor the development of a slowly evolving procedure in a system by analyzing dynamical responses of the system. The approach is applied and verified in different systems [22] including tracking fatigue in a mechatronic system [9]. Details about this approach can be found in Refs. [11, 23, 24]. For the convenience of illustration, the calculated GFDC is projected onto the ACPD signal using a least square fit as shown in Fig. 10(b). The two tracking results fit each other well. 


\section{Conclusions}

A brief introduction describing a novel fatigue testing rig was given. Compared with the traditional fatigue testing apparatus, this rig is able to duplicate arbitrary load histories with high forcing frequencies and various $R$ ratios. Using a three-step nonlinear system identification procedure, a numerical model was constructed to describe structural dynamics of the testing rig with undamaged specimen. Further analysis focused on the quantification of the nonlinearity, which showed that in practical testing scenarios the structural dynamics can be treated linear. The comparison of independently measured crack length and the corresponding generalized damage coordinate estimated from the measured structural oscillations was also shown based on some initial testing data.

\section{Acknowledgments}

This study was supported by the National Science Foundation Grants No. 0758536.

\section{References}

[1] Little, R., and Jebe, E., 1975. Statistical Design of Fatigue Experiments. Applied Science Publishers LTD, London.

[2] Stephens, R. I., Fatemi, A., Stephens, R. R., Fuchs, H. O., and Faterni, A., 2000. Metal Fatigue in Engineering. Wiley-Interscience;.

[3] Lee, Y.-L., Pan, J., Hathaway, R., and Barkey, M., 2004. Fatigue Testing and Analysis: Theory and Practice. Butterworth-Heinemann.

[4] Shawki, G. S., 1990. "A review of fatigue testing machines". Engineering Journal of Qatar University, 3, pp. 55-69.

[5] Weibull, W., 1960. Fatigue Testing And Analysis of Results. Advisory Group For Aeronautical Research and Development North Atlantic Treaty Organization.

[6] Bathias, C., 2006. "Piezoelectric fatigue testing machines and devices". International Journal of Fatigue, 28, pp. 1438-1445.

[7] Foong, C.-H., Wiercigroch, M., and F.Deans, W., 2006. "Novel dynamic fatigue-testing device: design and measurements". Measurement Science and Technology, 17, pp. 2218-2226.

[8] Chelidze, D., Cusumano, J., and Chatterjee, A., 2002. "Dynamical systems approach to damage evalution tracking, part i: Desccription and experimental application". Journal of Vibration and Acoutics, 124(2), pp. 250-257.

[9] Chelidze, D., and Liu, M., 2004. "Dynamical systems approach to fatigue damage identification". Journal of Sound and Vibration, 281, pp. 887-904.

[10] Chelidze, D., and Cusumano, J., 2006. "Phase space warping: Nonlinear time series analysis for slowly drifting systems". Philosophical Transactions of the Royal Society A,, 364, pp. 2495-2513.

[11] Chelidze, D., and Liu, M., 2008. "Reconstructing slowtime dynamics from fast-time measurements". Philosophical Transaction of the Royal Society A, 366, pp. 729-745.

[12] ASTM-E1820-08a, 2008. Standard Test Methods for Measurement of Fracture Toughness. Annual Book of ASTM Standards. American Society for Testing and Materials, Philadelphia, PA.

[13] Verboven, P., 2002. "Frequency-domain system identification for modal analysis". PhD thesis, Vrije Universiteit Brussel.

[14] Kerschen, G., Worden, K., Vakakis, A. F., and Golinval, J.-C., 2006. "Past, present and future of nonlinear system identification in structural dynamics". Mechanical Systems and Signal Processing, 20, pp. 505-592.

[15] Farrar, C. R., Cornwell, P. J., Doebling, S. W., and Prime, M. B., 2000. Structural health monitoring studies of the alamosa canyon and i-40 bridges. Tech. rep., Los Alamos National Laboratory.

[16] Farrar, C. R., Worden, K., Michael D. Todd and, G. P., Nichols, J., Adams, D. E., Bement, M. T., and Farinholt, K., 2007. Nonlinear system identification for damage detection. Tech. Rep. LA-14353, Los Alamos National Laboratory.

[17] Surace, C., Worden, K., and Tomlinson, G. R., 1992. "An improved nonlinear model for an automotive shock absorber". Nonlinear Dynamics, 3, pp. 413-429.

[18] Sibson, R., 1985. Manual for the TILE4 Interpolation Package. Department of Mathematics and Statistics, University of Bath.

[19] Olsson, H., Astrom, K. J., de Wit, C. C., Gafvert, M., and Lischinsky, P., 1998. "Friction models and friction compensation". Eur. J. Control, 4(3), pp. 176-195.

[20] Mohammad, K., Wordena, K., and Tomlinson, G., 1992. "Direct parameter estimation for linear and nonlinear structures". Journal of Sound and Vibration, 152, p. 3.

[21] Lewis, R. M., and Torczon, V., 1999. "Pattern search algorithms for bound constrained minimization". SIAM Journal on Optimization, 9, pp. 1082-1099.

[22] Dingwell, J., Napolitano, D., and Chelidze, D., 2006. "A nonlinear approach to tracking slow-time-scale changes in movement kinematics". Journal of Biomechanics, 40, pp. 1629-1634.

[23] Chelidze, D., and Liu, M., 2006. "Multidimensional damage identification based on phase space warping: An experimental study". Nonlinear Dynamics, 46(12), pp. 887-904.

[24] Chelidze, D., 2004. "Identifying multidimensional damage in a hierarchical dynamical system". Nonlinear Dynamics, 37(4), pp. 307-322. 